\title{
Neuro-fuzzy and nondominated sorting genetic algorithm based power system stabilizer design
}

\author{
Tawfik Guesmi* \\ College of Engineering, University of Hail, Hail, Saudi Arabia
}

\section{A R T I C LE IN F O}

\section{Article history:}

Received 8 January 2017

Received in revised form

21 March 2017

Accepted 27 April 2017

\section{Keywords:}

Power system stabilizer

Evolutionary algorithms

Non-dominated sorting genetic

algorithms

Adaptive neuro-fuzzy inference system

\begin{abstract}
A B S T R A C T
This study suggests a new method for online enhancement of multimachine system stability. Two steps centered on adjusting power system stabilizers (PSSs) are examined. Firstly, the PSS parameters are tuned off-line using an elitist optimization technique based on genetic algorithms symbolized by NSGAII over a large set of operating conditions. NSGAII was employed to move all electromechanical modes in a pre-specified area in the s-plan. Then, a flexible fuzzy logic-based neural network is proposed to adjust the parameters of the PSSs at any operating condition that can be outside the offline set by exploiting the off-line results. The suggested controllers are tested by using multi-machine system over some scenarios of serious faults and system configurations. Simulations results show the efficiency and sturdiness of the suggested stabilizers in enhancing the overall system dynamics in realtime at any loading condition selected arbitrarily.
\end{abstract}

(C) 2017 The Authors. Published by IASE. This is an open access article under the CC BY-NC-ND license (http://creativecommons.org/licenses/by-nc-nd/4.0/).

\section{Introduction}

In the past few years, power system problems associated with low frequency oscillations (LFO) have been widely revealed and examined. For economic and effectiveness reasons, power system stabilizers (PSSs) have been mostly applied along with excitation generator systems to improve both damping of LFO and stability of the power network during severe interferences. They are employed to develop damping torque for the rotor oscillations across the excitation systems. In most research works, the traditional PSS (CPSS) was commonly provided as a lead-lag filter (Li et al., 2009; Abido, 2000; Sebaa and Boudour, 2009). An extensive research to study the impact of the CPSS parameters on the temporary and powerful activities of power system is provided in (Kundur et al., 1989).

A quite a bit of research references have been released on the area of developing and applying of CPSSs controllers as sufficient damping resources (Ostojic, 1991; Sambariya et al., 2016). The design methods of PSSs such as phase compensation and root locus (Ostojic, 1991), eigenvalue sensitivity analysis (Tse et al., 2001) and poles placement (Abido, 2000) are very suggestive. In (Ataei et al.,

\footnotetext{
* Corresponding Author.

Email Address: tawfiq.guesmi@gmail.com

https://doi.org/10.21833/ijaas.2017.06.003

2313-626X/C) 2017 The Authors. Published by IASE.

This is an open access article under the CC BY-NC-ND license

(http://creativecommons.org/licenses/by-nc-nd/4.0/)
}

2012; Werner et al., 2003), the PSS adjustment issue is transformed into LMI problem; its solving determines the stabilizer parameters (Ataei et al., 2012). A new LMI based strategy with rank condition has been described in (Kim et al., 2010) to the design of effective PSS. Yet, a one machine test system is investigated in simulation section.

Unfortunately, some of the aforementioned techniques are sequential methods that only consider the damping improvement of one crucial electromechanical mode at a time. Moreover, they are repetitive techniques and require an initialization step. In addition, they can cause the convergence of the search process into local optima. The restrictions of traditional optimization methods were prevented by suggesting some soft computing algorithms. In Hassana et al. (2014), Sebaa and Boudour (2009), genetic algorithms were applied for effective PSS tuning. Two functions in accordance with the eigenvalue analysis have been developed for shifting all closed-loop electromechanical modes in a pre-specified zone in the s-plan. The multiobjective problem (MOP) is transformed into a mono-objective problem by assessing the goals with distinct weights. Accordingly, there is a loss of diversification in the Pareto solutions. In Sambariya et al. (2016), the authors provided a bat-based fuzzy algorithm for an effective PSS design where timedomain objective functions were employed. Other transformative calculations criteria and evolutionary computation algorithm, called bacteria foraging algorithm (BFA) along with maximum neuro-fuzzy 
plan were developed in Beno et al. (2011) and Mishra et al. (2007) to design intelligent adaptive flexible PSSs for the improvement of both the transient and dynamic stability of multi-machine systems. Nonetheless, The BFA depends on unique search guidelines which may lead to delay in attaining the global solution.

The above design procedures have been done either at single operating point or at several loading conditions at once. Unfortunately, the controllers cannot ensure the system stability with good performance, when loads and/or configuration of the system are changed. Thus, to solve such problem we have to update the controllers' parameters at each loading conditions, which does not guarantee the online decision. Recently, artificial intelligence based PSSs have been well-advised for online enhancement of stability (Fraile-Ardanuy and Zufiria, 2007; Lakshmi and Khan, 1998; Mitra et al., 2007; Abd-Elazim and Ali, 2013). A new neural network based technique for online CPSS setting has been provided in Segal et al. (2000). The stabilizer performance is investigated by varying terminal voltage, power generation and equivalent impedance of one generator system. In Fraile-Ardanuy and Zufiria (2007), genetic algorithms (GA) are combined with adaptive network based fuzzy inference systems (ANFIS) for online CPSS tuning. Unluckily, the above online designs are tested only by using a single generator system that cannot ensure the stability of a multimachine system. A fuzzy logic based procedure for online PSS design (FLPSS) was provided and analyzed in Lakshmi and Khan (1998) where speed deviations and their derivatives of machines were chosen as input signals. In Mitra et al. (2007), the FLPSS was used with new input signals, namely, deviation of the real power flow in some lines in combination with the speed deviation. This technique gives better results compared to the FLPSS with angular speed and acceleration input signals.

To use even the benefits of evolutionary algorithms, fuzzy logic systems and neural networks, a technique for real-time adjustment of the PSSs parameters using the improved version of nondominated sorting GA (NSGAII) (Deb et al., 2002; Guesmi et al., 2006) and ANFIS is suggested in this paper. The proposed online design will be done in two steps. The first stage aims to find the optimum PSS parameters using NSGAII for a lot of different operating conditions. Two objective functions will be minimized so as to shift as could as possible all undamped and lightly damped electromechanical modes in a pre-specified zone called D-shape area. In this research, the system will be linearized about each steady state condition.

Nevertheless, the next step relies on the ANFIS training phase according to the data base supplied by the previous step. Input and output data are respectively, operating conditions and optimum controller parameters. Optimum solution will be extracted from the Pareto front using the fuzzy based technique presented in Abido (2006). The suggested design approach ANFIS-PSS has been applied on a multi-machine power system under different scenarios of severe fault disturbances and loading. Eigenvalue analysis along with simulation results based on nonlinear simulation confirm that the suggested ANFIS-PSSs work effectively for any random operating condition and boosts on real-time the entire system performance.

The main contributions of this work are summarized as follows:

- A new NSGAII and ANFIS based PSS design for online enhancement of multimachine system stability was proposed. The aforementioned objective functions were minimized simultaneously and independently.

- Unlike previous works (Kashki et al., 2013; Khodabakhshian and Hemmati, 2013), the purpose of this study is not only to place all electromechanical modes in the D-shape area but also it aims to move them as much as possible to the left side.

The rest of this paper lies in six sections. In section two, a mathematical modeling of a multimachine system including PSSs is described. Section three investigates the mathematical formulation of the design of PSS controllers. NSGAII algorithm and the ANFIS principles are described respectively in sections four and five. Section six is consecrated to the establishment of the suggested controllers. A conclusion of this work is drawn in section seven.

\section{System modeling}

\subsection{Power system model}

For stability studies, power system is often modeled by nonlinear differential and algebraic equations (DAE) as follows (Fereidouni et al., 2013) (Eqs. 1-3).

$$
\begin{aligned}
& \dot{X}=f(X, Y, U) \\
& 0=g(X, Y) \\
& W=h(X, Y, U)
\end{aligned}
$$

Where, $X$ is the state vector defined by $X=$ $\left[\delta \omega E_{q}^{\prime} E_{f d}\right]^{T}, Y$ is the vector of algebraic variables composed of phase angles and magnitudes of voltages at all buses of the studied system and $W$ is the vector of output variables.

The PSS output signals constitute the input vector defined by $U . f$ is a vector function comprising the first order nonlinear differential equations that represent system and controller dynamics and $g$ describes the power flow equations in the network. $h$ is a vector function containing all equations that represent output variables.

A linear incremental model is incorporated in the PSS design method. By exploiting a methodology provided in Abd-Elazim and Ali (2013), power 
system with $n$ machines and $m$ PSSs is linearized at a steady state point as given below (Eq. 4).

$\left[\begin{array}{c}\Delta \dot{X} \\ 0 \\ \Delta W\end{array}\right]=\left[\begin{array}{lll}f_{x} & f_{y} & f_{u} \\ g_{x} & g_{y} & g_{u} \\ h_{x} & h_{y} & h_{u}\end{array}\right]$

where

$f_{x}=\nabla_{x}^{T} f, f_{y}=\nabla_{y}^{T} f, f_{u}=\nabla_{u}^{T} f, g_{x}=\nabla_{x}^{T} g g_{y}=\nabla_{y}^{T} g, g_{u}=$ $\nabla_{u}^{T} g, h_{x}=\nabla_{x}^{T} h, h_{y}=\nabla_{y}^{T} h$ and $h_{u}=\nabla_{u}^{T} h$

Removing $\Delta Y$ and assuming that power flow Jacobian $g_{y}$ is non-singular, the system can be readily modelled by the following state equation (Eq. 5).

$\dot{X}=A X+B U$

where, $A$ is $4 n \times 4 n$ matrix and $B$ matrix is $4 n \times m$ matrix that are described as follows:

$A=f_{x}-f_{y} g_{y}^{-1} g_{x}$ and $B=f_{u}-f_{y} g_{y}^{-1} g_{u}$

the output variables are written as follows (Eq. 6).

$\Delta W=C \Delta X+D \Delta U$

where $C=h_{x}-h_{y} g_{y}^{-1} g_{x}$ and $D=h_{u}-h_{y} g_{y}^{-1} g_{u}$.

\subsection{PSS with excitation system}

The PSS provides control effect through the exciter to the system under research. A lead-lag PSS portrayed in Fig. 1 is examined in this document (Abido, 2000).

The input signal of the $i$-th PSS is the normalized speed deviation, $\Delta \omega_{i}$. While the output is the supplementary stabilizing signal, $U_{i}$. As shown in the block diagram of Fig. 1, the PSS can be modelled by the transfer function given in Eq. 7 .

$U_{i}(s)=K_{i} \frac{s T_{W i}}{s T_{W i}}\left[\frac{\left(1+s T_{1 i}\right)\left(1+s T_{3 i}\right)}{\left(1+s T_{2 i}\right)\left(1+s T_{4 i}\right)}\right]$

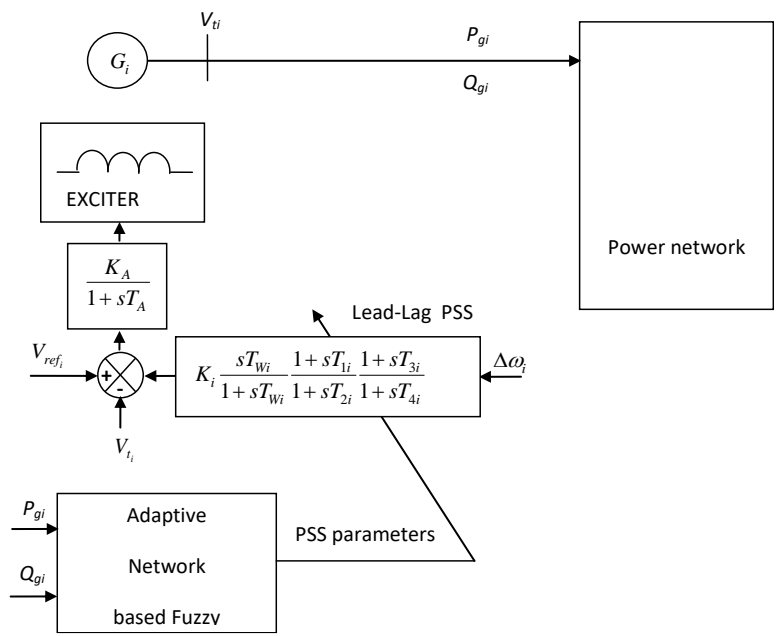

Fig. 1: Power network with ANFIS-PSS controllers
In the past equation, the washout block is used as a high-pass filter eventually continuous with time constant $T_{W i}$ sufficient to allow the signals in range 0.2-2 $\mathrm{Hz}$ associated with rotor oscillation to successfully pass without modification. In general, it is in the variety of 1-20s (Kundur et al., 1989). The two first order lead-lag filters are applied to compensate the phase lag between the output of the PSS and the control action that is the electric torque.

\section{Design strategy}

The closed-loop modes are analyzed after linearizing the system around the operating point. Then, objective functions will be formulated using only the lightly damped or unstable electromechanical modes that need to be shifted. In this document, the issue of PSS parameters tuning that consolidates the stability of the system is transformed into a bi-objective minimization issue. The first objective function indicated by $J_{1}$ in Eq. 8 aims to move all electromechanical mode into the left-side of the vertical line described by $\sigma_{i j}=\sigma_{0}$, Fig. $2 \mathrm{a}$. However, the second one is described by $J_{2}$. It is obvious that minimize $J_{2}$ matches to maximize the minimum of damping ratios of all electromechanical modes and place all electromechanical modes in a wedge-shape sector defined by $\xi_{i j} \geq \xi_{0}$, Fig. 2 b. As consequence, the maximum overshoot is limited. Compared with over previous works (Kashki et al., 2013; Khodabakhshian and Hemmati, 2013), the purpose of this formulation is not only to shift the electromechanical modes into the D-shape zone (Fig. 2c) but also it aims to move them as much as possible to the left side.

$$
\left\{\begin{array}{c}
\text { if } \sigma_{i j} \leq \sigma_{0} \text { and } \xi_{i j} \geq \xi_{0}, \\
J_{1}=\max \left(\sigma_{i j}\right) \\
J_{2}=-\min \left(\sigma_{i j}\right) \\
\text { else, } \\
J_{1}=J_{1 \max } \\
J_{2}=J_{2 \max }
\end{array}\right.
$$

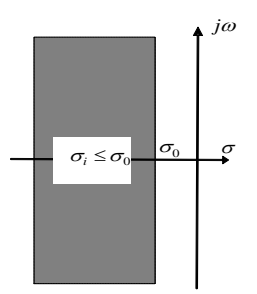

(a)

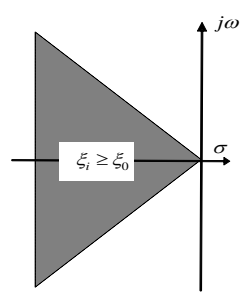

(b)

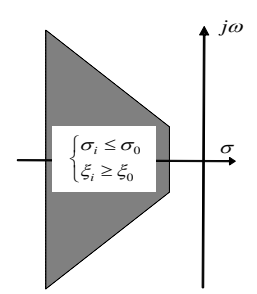

(c)
Fig. 2: Location of electromechanical modes

Objective functions $J_{1}$ and $J_{2}$ will be minimized with respect to the bounds of the PSSs parameters given by inequalities 9-13.

$$
\begin{aligned}
& K_{i}^{\min } \leq K_{i} \leq K_{i}^{\max } \\
& T_{1 i}^{\min } \leq T_{1 i} \leq T_{1 i}^{\max } \\
& T_{2 i}^{\min } \leq T_{2 i} \leq T_{2 i}^{\max } \\
& T_{3 i}^{\text {min }} \leq T_{3 i} \leq T_{3 i}^{\max } \\
& T_{4 i}^{\text {min }} \leq T_{4 i} \leq T_{4 i}^{\max }
\end{aligned}
$$


Other constraints can be included in system constraints, which are the electromechanical mode frequency limits (Eq. 14).

$\omega_{\min } \leq \omega \leq \omega_{\max }$

The time constant $T_{W i}$ of the washout will be fixed to $5 \mathrm{~s}$. Typical ranges of the decision variables are [1-20] for $K_{i}$ and [0.01-1.5] for $T_{4 i} . \omega_{\max }$ and $\omega_{\min }$ are 2 and $0.2 \mathrm{~Hz}$, respectively.

\section{Presentation of NSGAII approach}

As mentioned, NSGAII is employed in the optimization step. Starting by a population $P_{t}$ with $N_{p}$ individuals, NSGAII generates new population $Q_{t}$ called offspring population. These two populations will be combined in one population $R_{t}$. Then, $R_{t}$ is classified into different non-domination levels $R_{t}^{j}$. Thus, $R_{t}^{1}$ is the Pareto front. Generally speaking, all solutions of $R_{t}^{j}$ dominate all solutions of $R_{t}^{j+1}$. On the other hand, all solutions having the same nondomination level will be sorted in accordance with their crowding distances. Finally, solutions of $R_{t}^{1}$ are priority to be inserted into the next population $P_{t+1}$ followed by solutions from $R_{t}^{2}$ and so on until $P_{t+1}$ is filled. If the size of $P_{t+1}$ is greater than $N_{p}$, excess elements that are from the last front will be removed according to their crowding distances in ascending order. The basic flowchart of NSGAII is shown in Fig. 3.

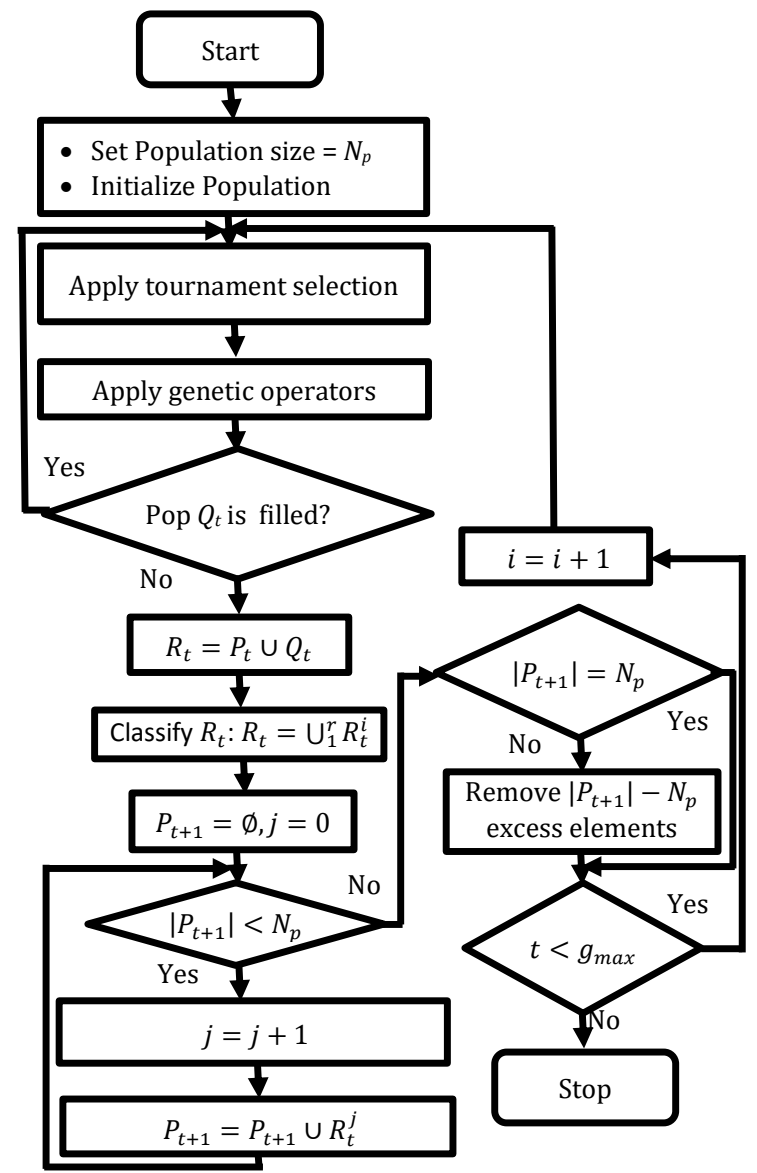

Fig. 3: Basic flowchart of NSGAII

\section{ANFIS technique}

ANFIS firstly proposed by Jang (1993) was used for online adjustment of PSSs parameters. The choice of the number of membership functions (MF) is a tricky step that influences the convergence of ANFIS method. Several works have been proposed to choose this parameter such as trial and error method and clustering based techniques (Fraile-Ardanuy and Zufiria, 2007). In this study, density measure based clustering approach is used in training phase. For a set $X=\left\{X_{1}, X_{2}, \cdots, X_{n}\right\}$ with $n$ data points, the density measure based clustering technique is summarized in Fig. 4. Where, $r_{a}$ and $r_{b}$ are positive constants that are set to 0.5 and 0.8 respectively.

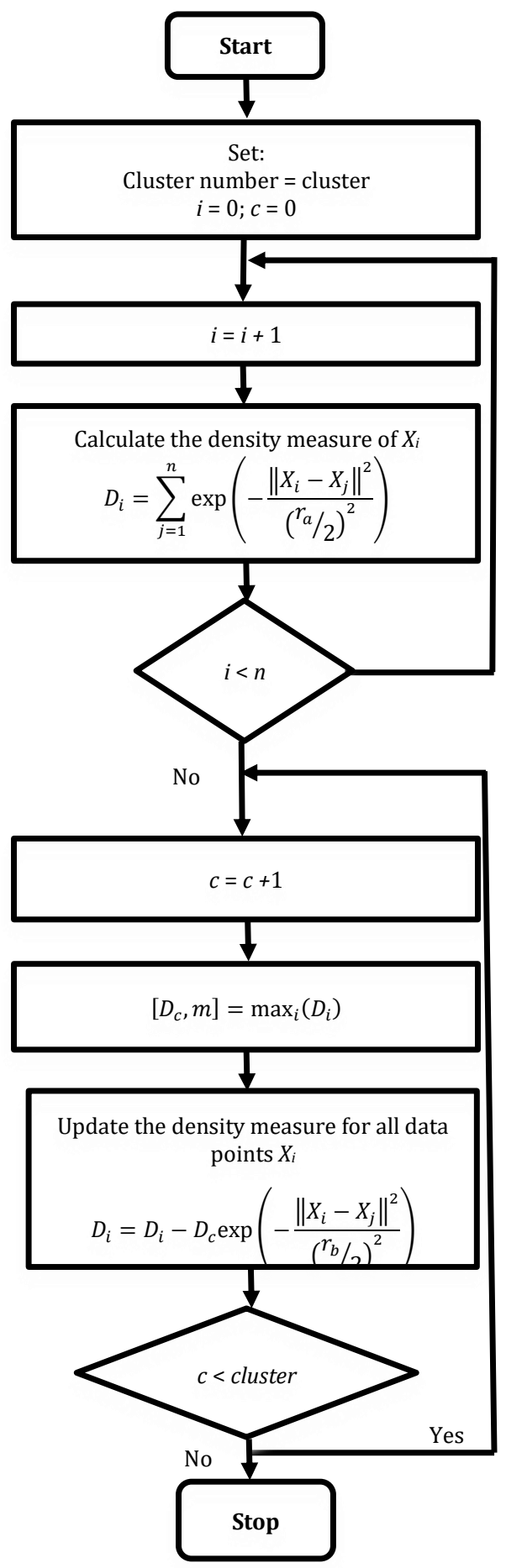

Fig. 4: Proposed clustering technique 
Fig. 5 illustrates an example of cluster points and cluster centers for two input variables that can be loading factors relative to active and reactive powers $P$ and $Q$. It is clear that the number of clusters is 8 for 1000 data points chosen randomly between 0.2 and 1.6 .

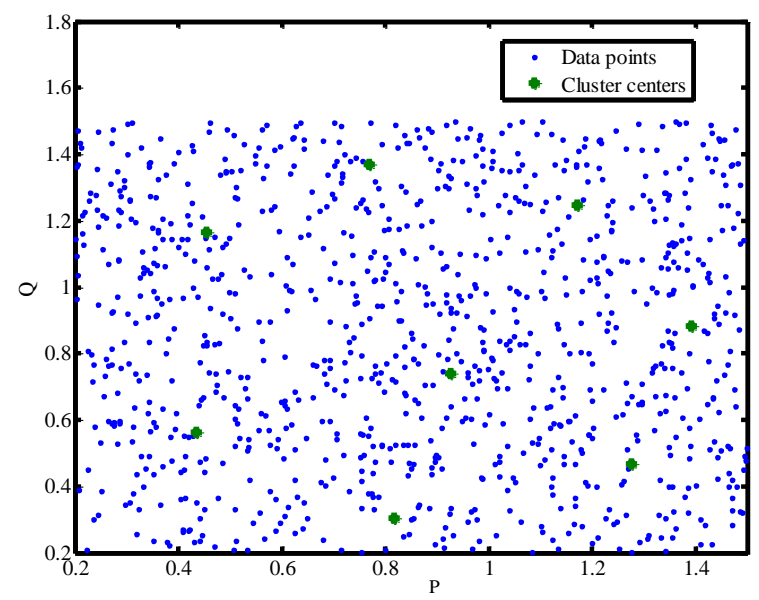

Fig. 5: Data points and cluster centers

\section{Implementation of the proposed controllers}

To illustrate the sturdiness and efficiency of the recommended ANFIS-based controllers (ANFIS-PSS), the 3-machine 9-bus WSCC (western system coordinating council) portrayed in Fig. 6 is regarded. All data of the studied system are presented in detail in Pai (1989). The system operating condition for the base case is depicted in Table 1. For this system, the assumption is that all machines except G1 are equipped with PSS. The pre-specified D-shape zone is defined by $\sigma_{0}=-2$ and $\xi_{0}=20 \%$.

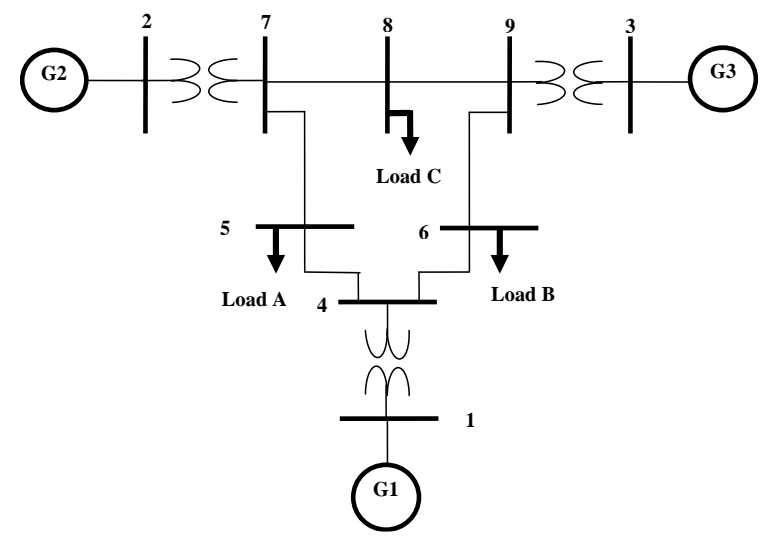

Fig. 6: WSCC system

Table 1: Operating condition for the base case

\begin{tabular}{cccc}
\hline & & $P[\mathrm{pu}]$ & $Q[\mathrm{pu}]$ \\
\hline \multirow{6}{*}{ Gen } & $\mathrm{G} 1$ & 0.72 & 0.27 \\
& $\mathrm{G} 2$ & 1.63 & 0.07 \\
& $\mathrm{G} 3$ & 0.85 & -0.11 \\
\multirow{4}{*}{ Load } & $\mathrm{A}$ & 1.25 & 0.50 \\
& $\mathrm{~B}$ & 0.90 & 0.30 \\
& $\mathrm{C}$ & 1.00 & 0.35 \\
\hline
\end{tabular}

\subsection{Off-line data collection}

In this stage, NSGAII algorithm was executed for preparing the input-output set. A large range of operating points described in Eqs. 23 and 24 are used. Where, $P_{L 0 i}$ and $Q_{L 0 i}$ correspond to the nominal case load of bus number $i$. Variables $\lambda_{p}$ and $\lambda_{q}$ are loading factors that will be varied individually, from 0.2 to 1.5 . In this manner, the load power variation ranges will be covered (Eqs. 15 and 16).

$P_{L i}=\lambda_{p} P_{L 0 i}$

$Q_{L i}=\lambda_{q} Q_{L 0 i}$

The set of training data is chosen to be with 1936 input-output pairs constituted by loading factors as input data and PSS parameters as output data.

To justify the use of NSGAII, a comparison with existing evolutionary algorithms such as NSGA is evident. For fair evaluation, NSGAII and NSGA programs have been written with the same number of generations (100), probability of crossover (0.9) and same probability of mutation (0.1).

The execution of the two methods is accomplished by generating the Pareto fronts depicted in Fig. 7, the variation of the fitness functions versus generation number (Fig. 8) and the optimized PSSs parameters illustrated by Table 2 . Obtained results for the base case show that NSGAII has better diversity characteristics with minimum values of fitness functions $J_{1}$ and $J_{2}$.

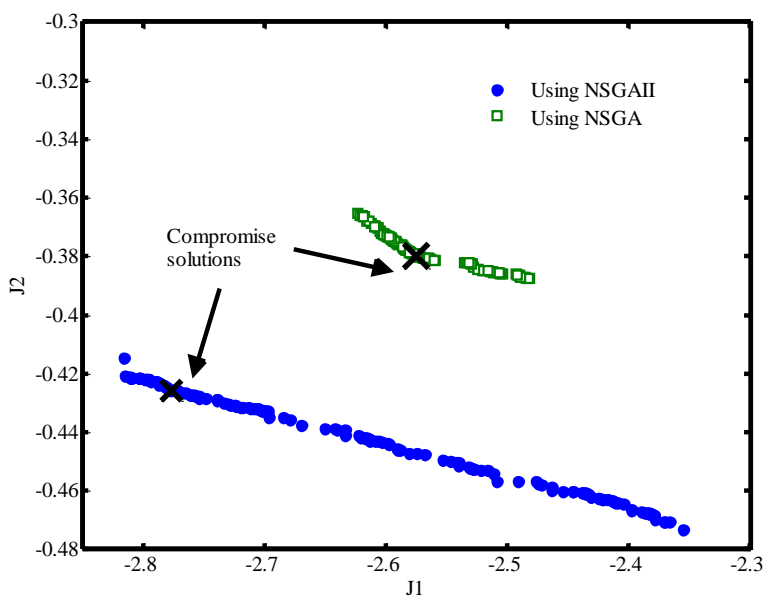

Fig. 7: Pareto solutions for the base case

Table 2: Optimal setting parameters

\begin{tabular}{ccccccc}
\hline Method & Gen. & $K$ & $T 1$ & $T 2$ & $T 3$ & $T 4$ \\
\hline \multirow{2}{*}{ NSGA } & G2 & 3.2940 & 0.0629 & 0.4234 & 1.4972 & 0.1046 \\
& G3 & 6.2904 & 0.2348 & 0.2235 & 0.2912 & 0.0210 \\
\multirow{2}{*}{ NSGAII } & G2 & 6.0874 & 0.9426 & 0.3129 & 0.2530 & 0.1352 \\
& G3 & 2.4439 & 1.4712 & 0.4607 & 0.1886 & 0.2000 \\
\hline
\end{tabular}

Electromechanical modes along with damping ratios at the base case without and with PSSs controllers are summarized in Table 3 . It is evident also, that the stabilizer NSGAII-PSSs outperforms the NSGA-PSSs and they provide the best damping. Moreover, there isn't any doubt that these modes are badly damped, when PSSs are not installed.

\subsection{Training phase}

The second stage of the online PSS design procedure aims to establish a relationship between 
input and output data by employing ANFIS. To accelerate the convergence of the learning stage, an ANFIS is applied for each controller parameter. Using the data base provided in the previous stage, the initial MFs with a cluster radius $r_{a}=0.5$ are displayed in Fig. 9. On the other hand, the performance of the ANFIS is tested by visualizing two parameters from its output set that are $K_{1}$ and $K_{2}$ for 44 samples from the checking set, Fig. 10.

Table 3: Electromechanical modes for base case

\begin{tabular}{cc}
\hline Method & Base case \\
\hline Without PSS & $-0.1124 \pm \mathrm{j} 7.7400,0.0145$ \\
NSGA-PSS & $-1.3346 \pm \mathrm{j} 9.1096,0.1450$ \\
(best compromise solution) & $-2.5748 \pm 5.6254 \mathrm{i}, 0.4162$ \\
NSGAII-PSS & $-2.7809 \pm 7.0134 \mathrm{i}, 0.3800$ \\
(best compromise solution) & $-2.8950 \pm 6.1314 \mathrm{i}, 0.5578$ \\
\hline
\end{tabular}
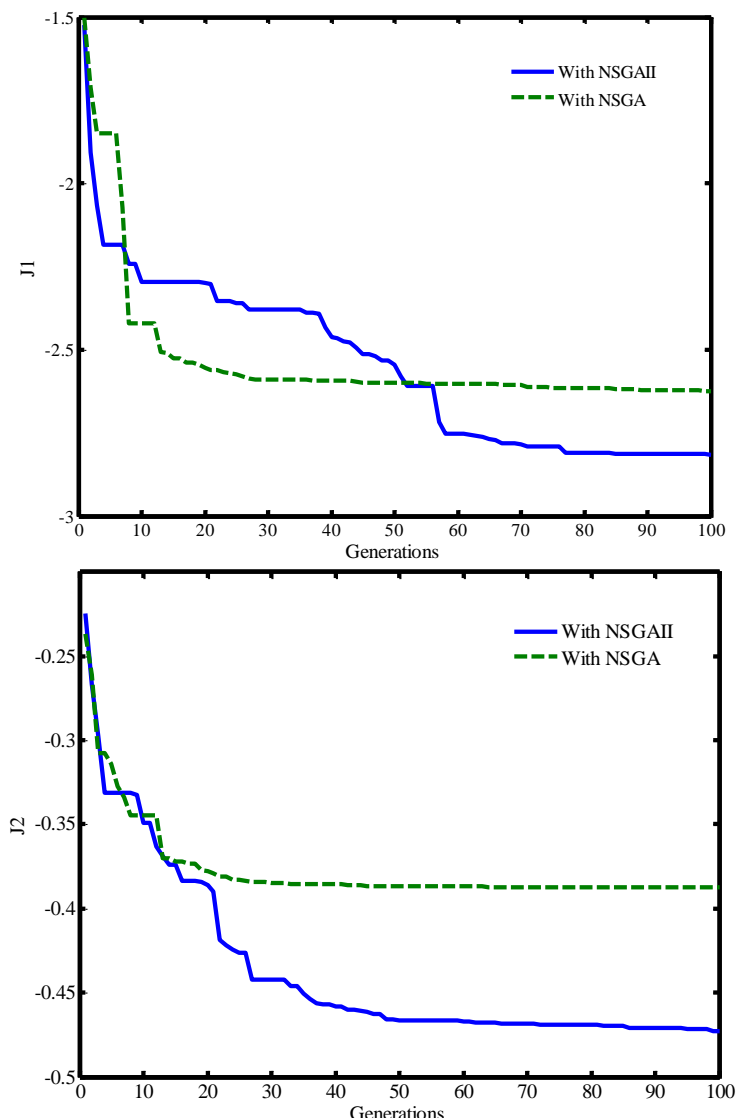

Fig. 8: Convergence of objective functions for the base case

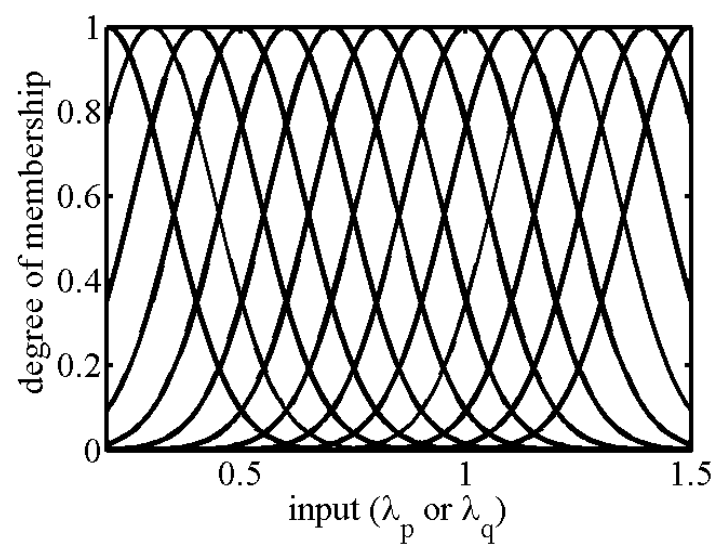

Fig. 9: Initial MF for PSSs parameters
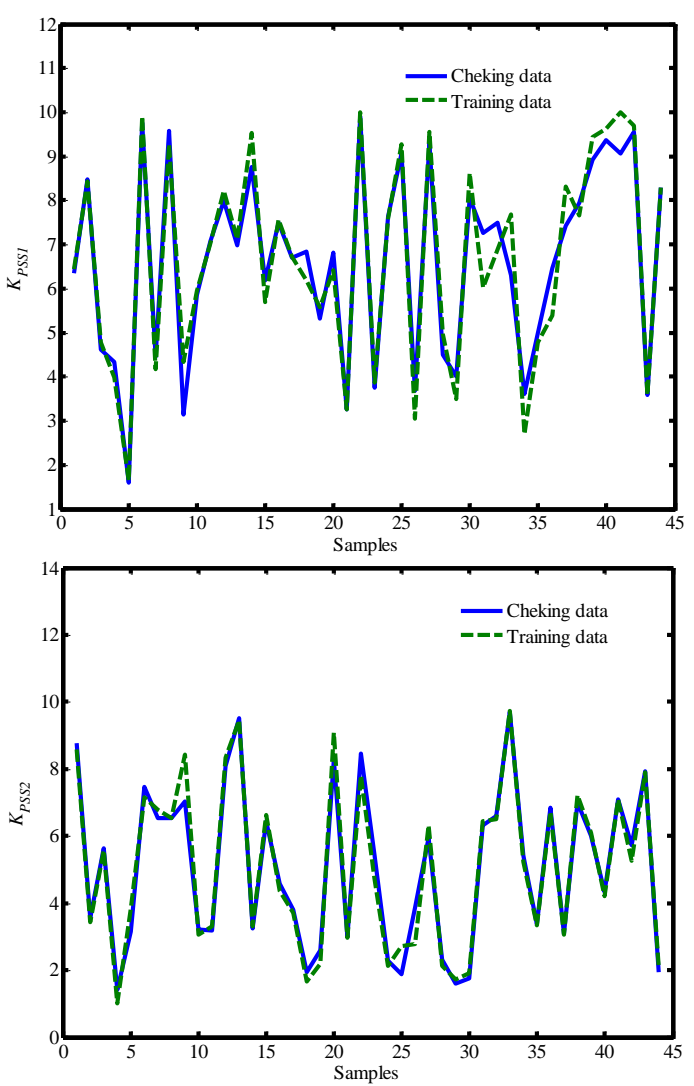

Fig. 10: Prediction data versus checking data

Fig. 11 indicates that all electromechanical modes for two operating conditions chosen arbitrary that are given in Table 4 have been properly shifted to the specified D-shape zone, when the suggested ANFIS-PSS controllers are installed.

In addition, it is obvious that these modes can be considered close to those achieved when applying the NSGAII based design. The first case correspond to $\lambda_{p}=0.4$ and $\lambda_{q}=0.7$. However, the second one is defined by $\lambda_{p}=0.2$ and $\lambda_{q}=1.1$.

\subsection{Nonlinear time-domain simulation}

The main purpose of this section is to confirm the efficiency of the ANFIS-PSS controllers in improving the system damping characteristics by nonlinear time-domain simulation. Therefore, two scenarios of severe fault disturbances are simulated for the two loading conditions mentioned above.

i) Scenario 1, 6-cycle fault disturbance at bus 5 at the end of line 5-7. The fault is cleared by tripping the line 5-7 with successful reclosure after 1.0s ;

ii) Scenario 2, is equivalent to the previous scenario with a step increase of 0.2 p.u. in mechanical power. The fault is removed by tripping the line 5-7 with successful reclosure after 1.0s.

Fig. 12 depicts some results obtained using nonlinear simulation at the previous arbitrary loading conditions. Since all system variables have the same dynamic performances, simply the speed deviations of generators are considered. It is obvious 
Table 4: Operating conditions for cases 1 and 2

\begin{tabular}{|c|c|c|c|c|c|}
\hline \multirow[b]{4}{*}{ Gen } & \multirow{4}{*}{$\begin{array}{l}\mathrm{G} 1 \\
\mathrm{G} 2\end{array}$} & \multicolumn{2}{|c|}{$\begin{array}{c}\text { Case } 1 \\
\lambda_{p}=0.4 \\
\lambda_{q}=0.7\end{array}$} & \multicolumn{2}{|c|}{$\begin{array}{c}\text { Case 2 } \\
\lambda_{p}=0.4 \\
\lambda_{p}=1.1\end{array}$} \\
\hline & & $P$ & $Q$ & $P$ & $Q$ \\
\hline & & 0.2864 & 0.1890 & 0.1432 & 0.2970 \\
\hline & & 0.6520 & 0.4690 & 0.3260 & 0.7370 \\
\hline & G3 & 0.3400 & $\begin{array}{c}- \\
0.7630\end{array}$ & 0.1700 & -0.1199 \\
\hline & A & 0.5000 & 0.3500 & 0.2500 & 0.5500 \\
\hline Load & B & 0.3600 & 0.2100 & 0.1800 & 0.3300 \\
\hline & $\mathrm{C}$ & 0.4000 & 0.2450 & 0.2000 & 0.3850 \\
\hline
\end{tabular}

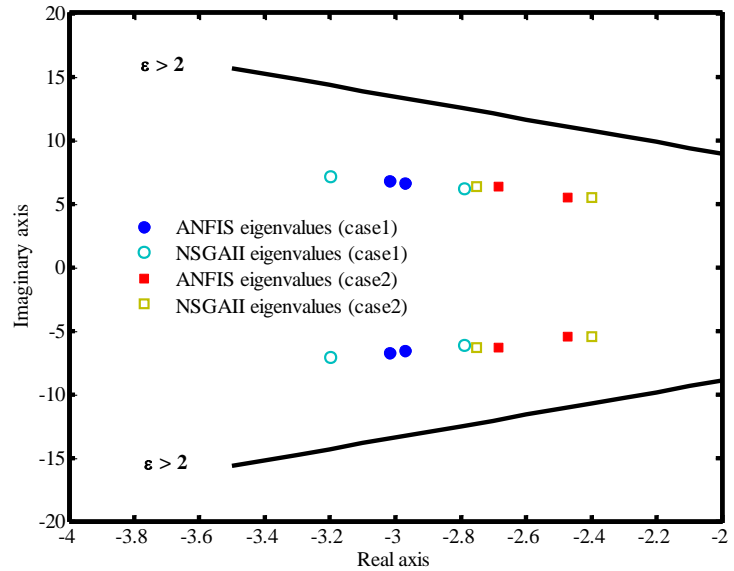

Fig. 11: D-shape sector for cases 1 and 2

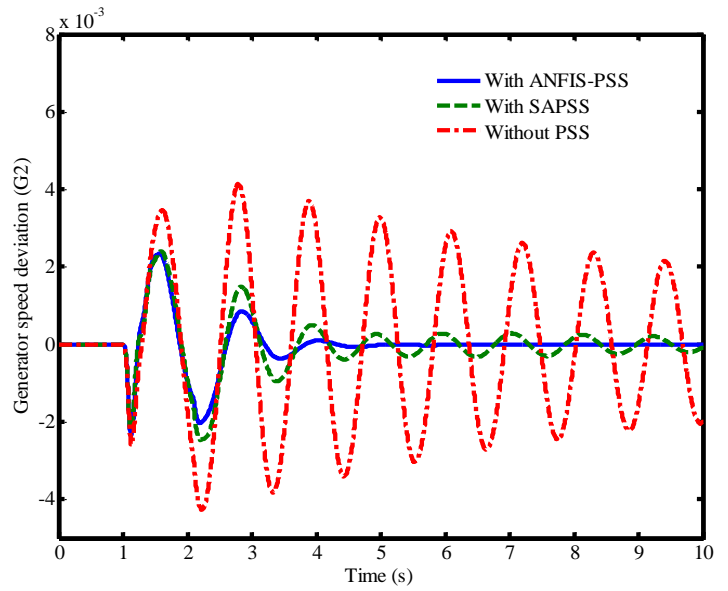

(a)

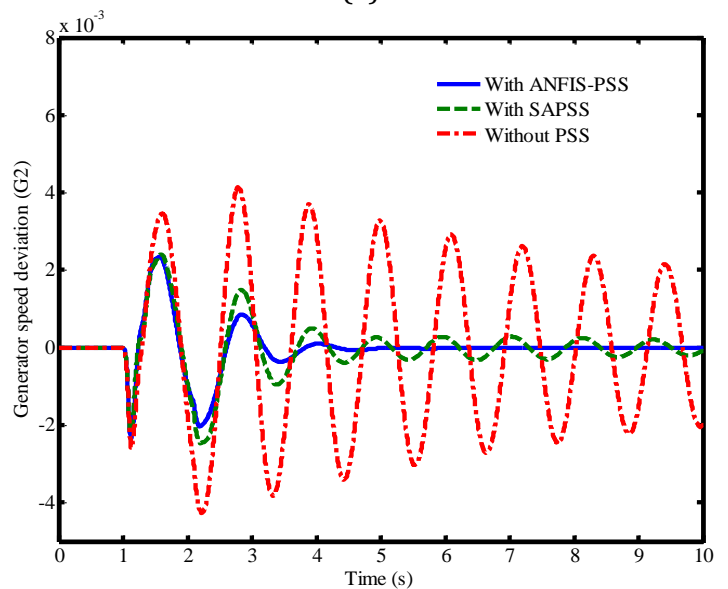

(b)

Fig. 12: Speed deviations in pu of G2 and G3 a) Scenario 1 for Case $1 \mathrm{~b}$ ) Scenario 2 for Case 2
Therefore, both controllers NSGAII-PSSs and ANFIS-PSSs lead to the same results. Nevertheless, Table 5 proves clearly that the recommended ANFISbased controllers are suitable for on-line stability enhancement due to its reduced CPU time. Results have been obtained using MATLAB R2009a installed on a PC with i7-4510U CPU @ 2.60 GHz, 64 bit.

Table 5: CPU time in seconds

\begin{tabular}{cc}
\hline NSGA-II algorithm & ANFIS-based approach \\
\hline 227.4479 & 0.0143 \\
\hline
\end{tabular}

Effectiveness of ANFIS-PSSs controllers is, also, verified and confirmed by two dynamic performance indices based on the generator speed deviation. The minimum values of these indices called ITAE and FD match an excellent damping. ITAE and FD are defined as follows (Eqs. 17 and 18).

$I T A E=\frac{\sum_{i=N g}^{n} 1000 \int_{0}^{t_{s i m}} t\left(\left|\Delta \omega_{i}\right|\right) d t}{N_{g}}$

$F D=\frac{\sum_{i=1}^{N g}\left(500 \times O S_{i}\right)^{2}+\left(500 \times U S_{i}\right)+T_{s i}^{2}}{N_{g}}$

where, $T_{S i}, U_{S i}$ and $O_{S i}$ are respectively, settling time, undershoot and overshoot of the $i$-th generator speed deviation $\Delta \omega_{i}$.

Form Figs. 13 and 14, it is clear that ANFIS-PSSs controllers have the best performance compared to NSGA-PSSs and the simulated annealing based power system stabilizer (SAPSS) proposed in (Abido, 2000).

\section{Conclusion}

This study attempts at suggesting a comprehensive scheme for online enhancement of power system stability. Firstly, an elitist technique based on GA symbolized by NSGAII is used to gather the training set. Real power and reactive power demands that can be directly measurable was considered as input data. However, optimum parameters of PSSs represent the output data. Then, the acquired data base is employed in the ANFIS training stage to provide the optimal stabilizer parameters at any loading condition.

The effectiveness of the suggested controllers has been tested on a well-known benchmark power system called "western system coordinating council". 

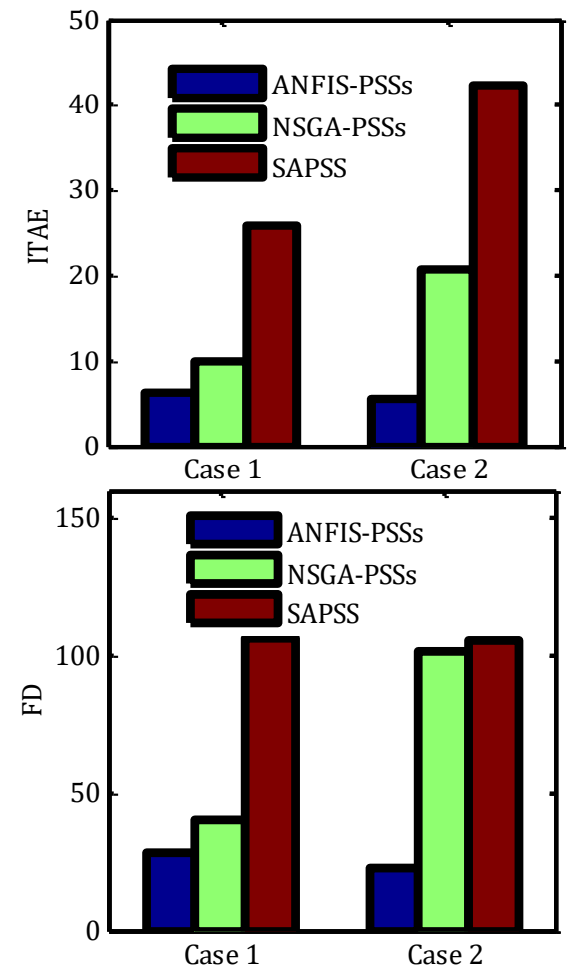

Fig. 13: Performance indices for Scenario 1

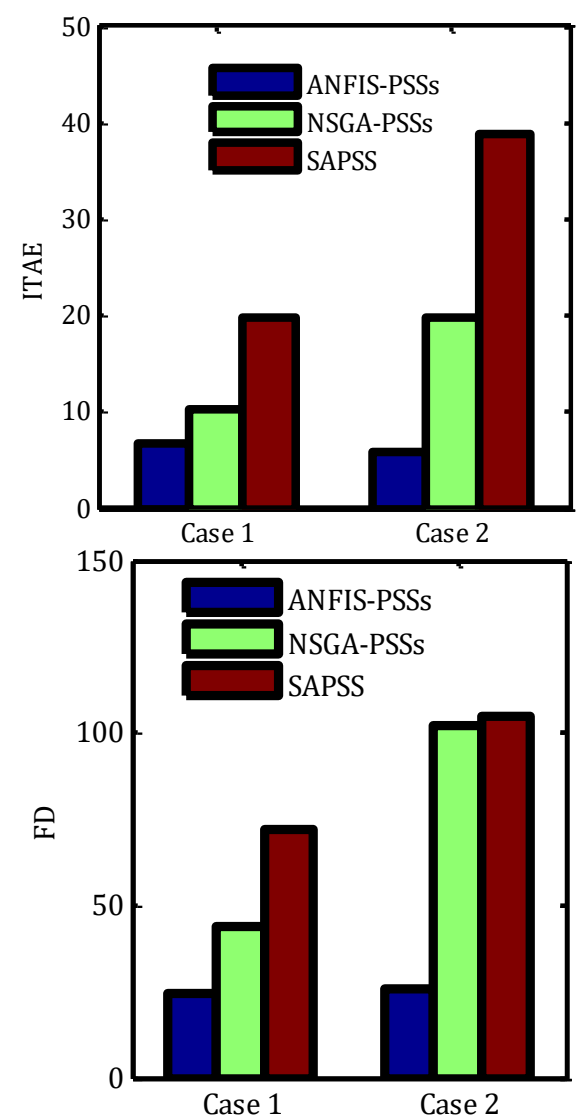

Fig. 14: Performance indices for Scenario 2

Simulation results approve the following outcomes:

- The proposed ANFIS-PSS controllers work effectively at any arbitrary operating condition and enhance the performance of the overall system on real-time.
- Since inputs are measurable variables, the suggested stabilizers can be implemented easily in practice.

\section{Acknowledgement}

The author would like to thank the Deanship of the Scientific Research of University of Hail, Saudi

Arabia for funding and supporting this research project (Project number: 0150410).

\section{List of symbols}

$\begin{array}{ll}E_{q}^{\prime} & \text { Internal voltage } \\ E_{f d} & \text { Field voltage } \\ V_{r e f i} & \text { Reference voltage of machine } G_{i} \\ V_{t i} & \text { Terminal voltage of machine } G_{i} \\ K_{A} & \text { Amplifier gain of the excitation system } \\ T_{A} & \text { Amplifier time constant of the excitation system } \\ T_{W i} & \text { Washout time constant } \\ g_{\max } & \text { Maximum number of generation } \\ J_{1} \text { and } J_{2} & \text { Fitness functions } \\ J_{1 \max } & \text { Upper limit of } J_{1} \\ J_{2 \max } & \text { Upper limit of } J_{2} \\ \delta & \text { Rotor angle of the machine } \\ \omega & \text { Speed deviation of the machine } \\ \sigma_{i j} & \text { Real part of the } i \text {-th electromechanical modes } \\ \end{array}$
corresponding to the $j$-th operating point

$\xi_{i j} \quad$ Damping ratio of the $i$-th electromechanical modes corresponding to the $j$-th operating point

$\sigma 0$ and $\xi_{0}$ Threshold parameters of the pre-specified $D$ shape sector

\section{References}

Abd-Elazim SM and Ali ES (2013). A hybrid particle swarm optimization and bacterial foraging for optimal power system stabilizers design. International Journal of Electrical Power and Energy Systems, 46: 334-341.

Abido MA (2000). Robust design of multimachine power system stabilizers using simulated annealing. IEEE Transactions on Energy Conversion, 15(3): 297-304.

Abido MA (2006). Multiobjective evolutionary algorithms for electric power dispatch problem. IEEE Transactions on Evolutionary Computation, 10(3): 315-329.

Ataei M, Hooshmand RA, and Parastegari M (2012). A wide range robust PSS design based on power system pole-placement using linear matrix inequality. Journal of Electrical Engineering, 63(4): 233-241.

Beno MM, Singh NA, Therase MC, and Ibrahim MMS (2011) Design of PSS for damping low frequency oscillations using bacteria foraging tuned non-linear neuro-fuzzy controller. In the GCC conference and exhibition, IEEE, Dubai, UAE: 653656. https://doi.org/10.1109/IEEEGCC.2011.5752639

Deb K, Pratap A, Agarwal S, and Meyarivan T (2002). A fast and elitist multiobjective genetic algorithm:NSGA-II. IEEE Transactions on Evolutionary Computation, 6(2): 182-197.

Fereidouni AR, Vahidi B, and Mehr TH, and Tahmasbi M (2013). Improvement of low frequency oscillation damping by allocation and design of power system stabilizers in the multimachine power system. International Journal of Electrical Power and Energy Systems, 52: 207-220.

Fraile-Ardanuy J and Zufiria PJ (2007). Design and comparison of adaptive power system stabilizers based on neural fuzzy networks and genetic algorithms. Neurocomputing, 70(16): 2902-2912. 
Guesmi T, Hadj Abdallah H, and Toumi A (2006). New approach to solve multiobjective environmental/economic dispatch. Journal of Electrical Systems, 2(2): 64-81.

Hassana LH, Moghavvemi M, Almurib HA, Muttaqi KM, and Ganapathy VG (2014). Optimization of power system stabilizers using participation factor and genetic algorithm. International Journal of Electrical Power and Energy Systems, 55: 668-679.

Jang JS (1993). ANFIS: Adaptive-network-base fuzzy inference system. IEEE Transactions on Systems, Man, and Cybernetics, 23(3): 665-685.

Kashki M, Abido MA and Abdel-Magid YL (2013). Power system dynamic stability enhancement using optimum design of pss and static phase shifter based stabilizer. Arabian Journal for Science and Engineering, 38(3): 637-650.

Khodabakhshian A and Hemmati R (2013). Multi-machine power system stabilizer design by using cultural algorithms. International Journal of Electrical Power and Energy Systems, 44(1): 571-580.

Kim SJ, Kwon S, and Moon YH (2010). Low-order robust power system stabilizer for single-machine systems: An LMI approach. International Journal of Control, Automation and Systems, 8(3): 556-563.

Kundur P, Klein V, Rogers GJ, and Zywno MS (1989). Application of power system stabilizers for enhancement of overall system stability. IEEE Transactions on Power Systems, 4(2): 614-626.

Lakshmi P and Khan MA (1998). Design of a robust power system stabilizer using fuzzy logic for a multi-machine power system. Electric Power Systems Research, 47(1): 39-46.

Li SY, Lee SS, Yoon YT, and Park JK (2009). Nonlinear adaptive decentralized stabilization control for multimachine power systems. International Journal of Control, Automation and Systems, 7(3): 389-397.
Mishra S, Tripathy M, and Nanda J (2007). Multimachine power system stabilizer design by rule based bacteria foraging. Electric Power Systems Research, 77(12): 1595-1607.

Mitra P, Chowdhury S, Pal S, Lahiri R, Song Y (2007). Performance of a fuzzy power system stabilizer with tie line active power deviation feedback. In the Conference on Power System and Exposition, IEEE, Atlanta, Georgia, USA: 884-889. https://doi.org/10.1109/PSCE.2006.296431

Ostojic DR (1991). Stabilization of multimodal electromechanical oscillations by coordinated application of power system stabilizers. IEEE Transactions on Power Systems, 6(4): 14391445.

Pai MA (1989). Energy function analysis for power system stability. Springer Science \& Business Media, Berlin, Germany.

Sambariya DK, Gupta R, and Prasad R (2016). Design of optimal input-output scaling factors based fuzzy PSS using bat algorithm. Engineering Science and Technology, an International Journal, 19(2): 991-1002.

Sebaa K and Boudour M (2009). Optimal locations and tuning of robust power system stabilizer using genetic algorithms. Electric Power Systems Research, 79(2): 406-416.

Segal R, Kothari ML and Madnani S (2000). Radial basis function (RBF) network adaptive power system stabilizer. IEEE Transaction on Power Systems, 15(2): 722-727.

Tse CT, Wang KW, Chung CY, and Tsang KM (2001). Robust PSS design by probabilistic eigenvalue sensitivity analysis. Electric Power Systems Research, 59(1): 47-54.

Werner H, Korba P, and Yang CT (2003). Robust Tuning of Power System Stabilizers Using LMI-Techniques. IEEE Transactions on Control Systems Technology, 11(1): 147-152. 